\title{
Pleomorphic Adenoma of the Lateral Nasal Wall: An Unusual Entity
}

\author{
${ }^{1}$ Apoorva K Pandey, ${ }^{2}$ Aparna Bhardwaj, ${ }^{3}$ Tripti Maithani, ${ }^{4}$ Sonam Rathi
}

\begin{abstract}
Pleomorphic adenomas occurring in the nasal cavity are rarely encountered; nevertheless, the abundance of minor seromucinous glands in that region is seen. Most cases present with a painless, slowly progressing unilateral nasal obstruction, a nasal mass, and epistaxis. Herewith, we present an unusual case of pleomorphic adenoma arising from the lateral wall of right nasal cavity in a 41-year-old male, who complained of right nasal obstruction with no history of epistaxis. Rigid nasal endoscopy revealed a swelling continuous with right inferior concha. Computed tomography showed a well-defined, soft tissue mass in the right nasal cavity. The lesion was completely excised endoscopically. Histopathology confirmed a pleomorphic adenoma consisting of epithelial and mesenchymal elements. Wide exposure is crucial for complete excision with negative margins to achieve positive outcome and to prevent recurrence.
\end{abstract}

Keywords: Endoscopic excision, Lateral nasal wall, Pleomorphic adenoma.

How to cite this article: Pandey AK, Bhardwaj A, Maithani T, Rathi S. Pleomorphic Adenoma of the Lateral Nasal Wall: An Unusual Entity. Clin Rhinol An Int J 2017;10(1):11-13.

Source of support: Nil

Conflict of interest: None

\section{INTRODUCTION}

Pleomorphic adenomas (benign mixed tumor) are the most common salivary gland tumors, and the majority of them occur in the parotid gland followed less frequently in the minor salivary glands. Minor salivary gland tumors can occur anywhere in upper aerodigestive tract. These lesions have been reported in the nasal cavity, paranasal sinuses, nasopharynx, oropharynx, hypopharynx, and larynx. In the upper respiratory tract, the most common site is the nasal cavity followed

\footnotetext{
${ }^{1}$ Associate Professor, ${ }^{2,3}$ Professor, ${ }^{4}$ Senior Resident

${ }^{1,3,4}$ Department of ENT, Shri Guru Ram Rai Institute of Medical \& Health Sciences, Dehradun, Uttarakhand, India

${ }^{2}$ Department of Pathology, Shri Guru Ram Rai Institute of Medical \& Health Sciences, Dehradun, Uttarakhand, India

Corresponding Author: Apoorva K Pandey, Associate Professor, Department of ENT, Shri Guru Ram Rai Institute of Medical \& Health Sciences, Dehradun, Uttarakhand, India Phone: +9101352760439, e-mail: apoorva.pande@yahoo.co.in
}

by maxillary sinus and the nasopharynx. ${ }^{1}$ The first ever reported case in the literature of a pleomorphic adenoma of nasal cavity dates back to $1929 .^{2}$ The larger case series of intranasal pleomorphic adenomas have been reported by Spiro et $\mathrm{al}^{3}$ with 40 cases and Compagno and Wong ${ }^{4}$ with 40 cases. As the name suggests, these tumors exhibit a very diverse histologic appearance containing both epithelial and mesenchymal tissue components. We present an unusual case of pleomorphic adenoma of the lateral wall of nasal cavity.

\section{CASE REPORT}

A 41-year-old man, nonsmoker and nonalcoholic, presented at the otolaryngology department with complaints of progressively increasing right nasal obstruction and a right nasal mass for last 2 years. He had no other complaints, such as epistaxis, anosmia, facial pain, sneezing, nasal discharge, or external nasal swelling. Anterior rhinoscopy showed a smooth, pink-whitish, translucent mass in right nasal cavity with septum deviated to the left, and on probing, it could be passed all around the lesion except in its lateral relation. This painless mass did not bleed on touch, had no change in size after decongestion, and a needle aspiration demonstrated a dry tap. Rigid nasal endoscopy revealed a large polypoidal mass present in the right nasal cavity, which was continuous with inferior turbinate and lined by normal looking mucosa. No other ear, nose, throat, and neck abnormality was found on clinical examination. Computed tomography (CT) scan revealed a polypoidal soft tissue mass measuring $5.4 \times 1.9 \times 3.8 \mathrm{~cm}$ in the right nasal cavity. Right middle turbinate was not defined and rarefaction of inferior concha and obliteration of osteomeatal complex was seen. Medial wall of right maxillary sinus was remodeled and displaced laterally. Nasal septum was deviated toward left side (Figs $1 \mathrm{~A}$ to $\mathrm{C}$ and 2). Intranasal endoscopic excision under general anesthesia was done along with taking $1 \mathrm{~cm}$ margin of normal adjacent mucosa around lesion. The patient was discharged after 3 days, and the postoperative period was uneventful. The patient is under regular follow-up.

Histopathology report of the tumor showed a mixed epithelial and mesenchymal appearance (Fig. 3). Epithelial component was seen in the form of ducts, tubules, 

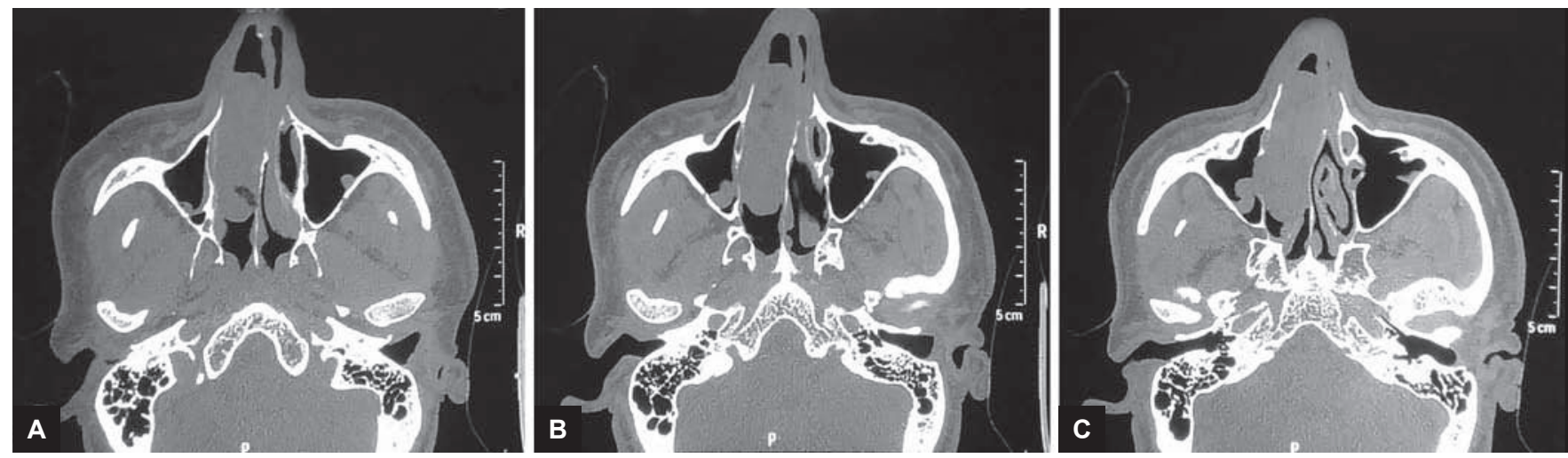

Figs 1A to C: Computed tomography scan (axial cuts) shows polypoidal mass in right nasal cavity

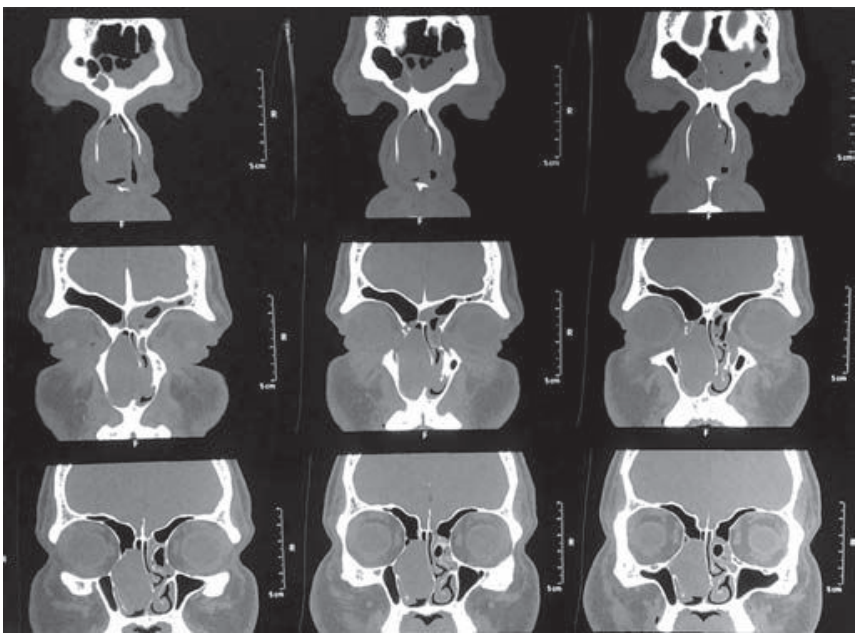

Fig. 2: Computed tomography scan (coronal cuts) reveals polypoidal soft tissue mass measuring $5.4 \times 1.9 \times 3.8 \mathrm{~cm}$ in right nasal cavity. Right middle turbinate was not defined and rarefaction of inferior concha and obliteration of osteomeatal complex was seen

acini, strands, and sheets, which were dispersed in the chondromyxoid stroma, and this report was consistent with the diagnosis of pleomorphic adenoma.

\section{DISCUSSION}

Pleomorphic adenomas occurring in the nasal cavity are extremely unusual. They present between the third and sixth decade, and are seen predominantly in females. Its correlation with occupational exposure or inhaled toxic chemicals has not been reported. It has a slowgrowing course and, hence, clinical features manifest after a long time. ${ }^{5}$ Clinical features include unilateral nasal obstruction, epistaxis, and presence of a nasal mass and mucopurulent nasal discharge. Other features like external swelling of nasal pyramid and pain, when it enlarges, can also occur. ${ }^{5}$

The serous and mucous glands are abundant in the lateral nasal wall; nevertheless, pleomorphic adenomas of the nasal cavity more commonly arise from the nasal septum. Different theories have been suggested to unfold this fact. Stevenson ${ }^{6}$ elucidated that remnants of

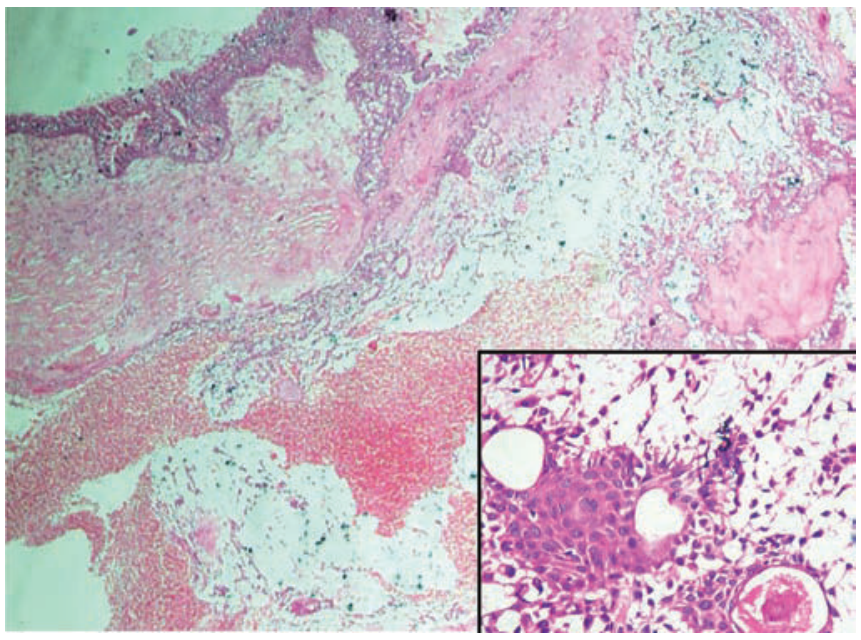

Fig. 3: Histopathology showing (100x, hematoxylin and eosin stain) tissue composed of epithelial and mesenchymal elements. Inset shows epithelial element in the form of ducts, tubules, acini, strands, and sheets, which are dispersed in chondromyxoid stroma (400x)

vomeronasal organ, an epithelium-lined duct in cartilaginous septum degenerated in early intrauterine period, could be the reason for occurrence of this tumor in that specific region. ${ }^{6}$ Ersner and Saltzman ${ }^{7}$ proposed that the precursors of the septal pleomorphic adenomas are ectopic embryonic epithelial cells on the septal mucosa, found during the migration of the nasal buds. ${ }^{7}$

Pleomorphic adenomas are defined by epithelial tissue mixed with components of myxoid, mucoid, or chondroid tissue. Histologically, pleomorphic adenoma of the aerodigestive tract may mimic aggressive epithelial tumors due to high cellularity and lack of a stromal component. Immunohistochemistry favors positive on staining for various cytokeratins, S100 protein, glial fibrillary acid protein, vimentin, a smooth muscle actin. ${ }^{5}$

Pleomorphic adenomas of the nasal cavity possess peculiar cytomorphologic features, which characteristically differentiates them from pleomorphic adenomas of the parotid gland. Cellularity is, generally, greater in pleomorphic adenomas of the nasal cavity as compared with those arising in major salivary glands. ${ }^{4} \mathrm{On} C \mathrm{CT}$, 
pleomorphic adenomas may look nonhomogeneous due to mesenchymal stroma, cystic degeneration, or necrosis, whereas nasal pleomorphic adenomas appear homogeneous owing to their high cellularity. ${ }^{8}$ On magnetic resonance imaging, T1-weighted images show homogeneous mass lesion isointense to brain, and fast-spin echo short inversion time inversion recovery images heterogeneous with high signal intensity. ${ }^{9}$

Differential diagnosis of intranasal pleomorphic adenoma includes squamous cell carcinoma, adenocarcinoma, adenoid cystic carcinoma, mucoepidermoid carcinoma, melanoma, olfactory esthesioneuroblastoma, polyps, papillomas, angiofibroma, and osteoma. ${ }^{5}$

The treatment of choice is complete surgical excision with wide tumor-free margins. It is important to discuss here that these tumors always carry the risk of recurrence irrespective of their site of origin. Wide exposure with complete excision dictates the final outcome. The surgical approach taken depends on the size, location, extent of the lesion, and associated conditions, such as presence of sinusitis or deviated septum. Various approaches described in literature include intranasal, transnasal endoscopic, external rhinoplasty, lateral rhinotomy, and midfacial degloving. ${ }^{10}$ Recurrence can also occur, though infrequent. Compagno and Wong ${ }^{4}$ reported three cases of local recurrence in his series of 40 cases $(7.5 \%)$. ${ }^{4}$ Postoperative radiotherapy has been recommended by some authors in cases where recurrence occurred. ${ }^{11}$

A neoplasm arising from the nasal septum carries a higher potential of malignancy compared with other sites in the nose. ${ }^{12}$ At times, pleomorphic adenoma may express malignant transformation, the most common type being carcinoma ex pleomorphic adenoma, which has a tendency to metastasize to bone, lung, lymph nodes, and liver. ${ }^{13}$

\section{CONCLUSION}

Pleomorphic adenoma of the nasal cavity is an exceedingly rare clinical entity. It presents a difficult and even perplexing diagnostic problem because of similar clinical features of other nasal tumors. The treatment of choice is wide excision with negative tumor margins. Wide exposure is crucial to achieve complete removal of the tumor. A regular postoperative examination in follow-up visits is essential owing to its potential for recurrence.

\section{REFERENCES}

1. Gana P, Masterson L. Pleomorphic adenoma of the nasal septum: a case report. J Med Case Rep 2008 Nov;2:349.

2. Denker A, Kahler O. Handush Der Hals. Nasen ohrenheilkunde 1929;5:202.

3. Spiro RH, Koss LG, Hadjdu SL. Tumours of minor salivary origin. A clinicopathologic study of 492 cases. Cancer 1973 Jan;31(1):117-129.

4. Compagno J, Wong RT. Intranasal mixed tumours (pleomorphic adenomas): a clinicopathologic study of 40 cases. Am J Clin Pathol 1977 Aug;68(2):213-218.

5. Sciandra D, Dispenza F, Porcas R, Kulamarva G, Saraniti C. Pleomorphic adenoma of the lateral nasal wall: case report. Acta Otorhinolaryngol Ital 2008 Jun;28(3):150-153.

6. Stevenson HN. Mixed tumour of the septum. Ann Otol Rhinol Laryngol 1932 Jun;41:563-570.

7. Ersner MJ, Saltzman M. A mixed tumour of the nasal septum. Report of a case. Laryngoscope 1944 Jun;54(6):287-296.

8. Ozturk E, Saglam O, Sonmez G, Cuce F, Haholu A. CT and MRI of an unusual intranasal mass: pleomorphic adenoma. Diagn Interv Radiol 2008 Dec;14(4):186-188.

9. Motoori K, Takano H, Nakano K, Yamamoto S, Ueda T, Ikeda M. Pleomorphic adenoma of the nasal septum: MR features. AJNR Am J Neuroradiol 2000 Nov-Dec;21(10):1948-1950.

10. Avishay G, Yudith B, Fradis Milo. Pleomorphic nasoseptal adenoma. J Otolaryngol 1997 Dec; 26(6):399-401.

11. Mackie T, Zahirovic A. Pleomorphic adenoma of the nasal septum. Ann Otol Rhinol Laryngol 2004 Mar;113(3): 210-211.

12. Rauchfuss A, Stadtler F. The differential diagnosis of benign neoplasm of the nasal septum. HNO 1981 Apr;29(4): 124-127.

13. Freeman FB, Kennedy KS, Parker GS, Tatum SA. Metastasizing pleomorphic adenoma of the nasal septum. Arch Otolaryngol Head Neck Surg 1990 Nov;116(11):1331-1333. 